\section{Versand von Proben}

\author{
W. G. Guder \\ München, Deutschland
}

Synonym(e) Blutversand; Probenversand; Postversand von Proben; Urinversand

Englischer Begriff mailing samples; posting samples

Definition Vorschriften, die beim Versand von diagnostischen Proben über Land, in der Luft und mit der Post zu beachten sind.

Beschreibung Beim Versand von diagnostischen oder anderen biologischen oder medizinischen Untersuchungsgütern sind die Vorschriften bezüglich der Verpackung und Beschriftung zu beachten. Die Absender von Untersuchungsgut ( $\triangleright$ Untersuchungsgut, biologisches) müssen sicherstellen, dass die Sendungen derart verpackt sind, dass sie den Bestimmungsort in gutem Zustand erreichen und während des Transports keinerlei Gefahr für Mensch, Tier und Umwelt darstellen. Bei Sendungen von infektiösem Material muss auf der Aufschriftseite links neben der Anschrift der auffällige Vermerk „Untersuchungsgut - Vorsicht infektiös“ angebracht sein. Für grenzüberschreitenden Verkehr ist diese Aufschrift in französischer Sprache „Matières Biologiques Perissables“ erforderlich. Die haftrechtlichen Folgen trägt grundsätzlich der Absender. Das Versand- und Transportwesen akzeptiert nur Verpackungen, die folgendem Inhalt entsprechen:
- Innenverpackung für das Untersuchungsgut

- Aufsaugendes Material

- Außenverpackung als Schutzgefäß für das Probengefäß

- Versandhülle, die mit den entsprechenden Zeichen für medizinisches oder biologisches Untersuchungsgut bezeichnet sind

Über Mehrverpackungen in Kisten und Metallbehältern informiert die Gefahrstoffverordnung Straße und Eisenbahn GGVS oder GGVE ( $\triangleright$ Gefahrstoffpiktogramme).

Dazu gibt es noch ein allgemeines Zeichen für „Gefahr durch biologisches Material“, das auch beim Versand von menschlichem Untersuchungsmaterial anzubringen ist. Dies ist durch Europäische Gesetzgebung einheitlich in Europa gültig.

\section{Literatur}

Deutsche Post AG (2010) Regelungen über den Postversand von medizinischem und biologischem Untersuchungsgut. http://www.suesse.de/ service/postversand-medizinisches-biologisches-untersuchungsgut/ 2017

EN 829 (1996) In vitro diagnostic systems. Transport packages for medical and biological specimens. Requirements, tests. European Committee for Standardization (CEN), Brüssel

Guder WG, Narayanan S (Hrsg) (2015) Sample transport, treatment after arrival, storage and disposal. In: Preexamination Procedures in Laboratory Medicine. Berlin/Boston: Walter de Gruyter, S 251-263

Thurm V, Tschäpe H (2001) Gefahrgutrechtliche Voraussetzungen für den Versand von Diagnostischen Proben, Bakterienkulturen u. a. infektiösen Materialien. Bundesgesundheitsbl. Gesundheitsforsch Gesundheitsschutz 44:823-828

United Nations (2005) Recommendations on the transport of dangerous goods. Model regulations, 14th rev edn. UN, New York/Geneva 